\title{
Seroprevalence of bovine theileriosis in northern China
}

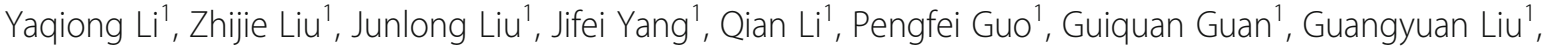 \\ Jianxun Luo ${ }^{1 *+}$, Hong Yin ${ }^{1,2^{* \dagger}}$ and Youquan Li ${ }^{1{ }^{*+}}$
}

\begin{abstract}
Background: Bovine theileriosis is a common disease transmitted by ticks, and can cause loss of beef and dairy cattle worldwide. Here, an indirect enzyme-linked immunosorbent assay (iELISA) based on Theileria luwenshuni surface protein (TISP) was developed and used to carry out a seroepidemiological survey of bovine theileriosis in northern China.
\end{abstract}

Methods: We used the BugBuster Ni-NTA His•Bind Purification Kit to purify recombinant TISP (rTISP), which was subsequently analyzed by Western Blotting to evaluate cross-reactivity with other pathogen-positive sera. The iELISA method based on rTISP was successfully developed. Sera from 2005 blood samples were tested with the rTISP-iELISA method, and blood smears from these samples were observed by microscopy.

Results: The specificity of iELISA was $98.9 \%$, the sensitivity was $98.5 \%$, and the cut-off was selected as $24.6 \%$. Western Blot analysis of rTISP confirmed that there were cross-reactions with Theileria luwenshuni, Theileria uilenbergi, Theileria ovis, Theileria annulata, Theileria orientalis and Theileria sinensis. The epidemiological survey showed that the highest positive rate of bovine theileriosis was $98.3 \%$, the lowest rate was $84.1 \%$, and the average positive rate was $95.4 \%$ by iELISA. With microscopy, the highest positive rate was $38.9 \%$, the lowest rate was $5.1 \%$, and the relative average positive rate was $13.7 \%$.

Conclusions: An rTISP-iELISA was developed to detect circulating antibodies against bovine Theileria in northern China. This is the first report on the seroprevalence of bovine theileriosis in northern China, and it also provides seroepidemiological data on bovine theileriosis in China.

Keywords: ELISA, Microscopy, Prevalence, Theileriosis

\section{Background}

Bovine theileriosis is a constraint to the cattle industry in many developing countries because it causes morbidity and mortality in calves and exotic cattle [1-3]. Generally, indigenous cattle have developed resistance to ticks and tick-borne pathogens in endemic areas. However, the susceptibility of exotic breeds presents a major obstacle to the improvement of cattle production and breeding [4]. The causative agents of bovine theileriosis

\footnotetext{
* Correspondence:

luojianxun@caas.cn; yinhong@caas.cn; youquan-li@163.com

†Equal contributors

${ }^{1}$ State Key Laboratory of Veterinary Etiological Biology, Key Laboratory of Veterinary Parasitology of Gansu Province, Lanzhou Veterinary Research Institute, Chinese Academy of Agricultural Science, Xujiaping 1, Lanzhou, Gansu 730046, People's Republic of China

Full list of author information is available at the end of the article
}

reported in China are Theileria annulata, Theileria orientalis and Theileria sinensis [3, 5-7]. Five kinds of ticks, namely, Hyalomma detritum, Hyalomma anatolicum anatolicum, Haemaphysalis longicornis, Haemaphysalis qinghaiensis and Haemaphysalis japonica, can transmit ovine and bovine Theileria infection in China [8]. Of them, Hya. detritum and Hya. anatolicum anatolicum can transmit T. annulata [9], and Hya. anatolicum anatolicum can also transmit Theileria ovis [10]; Haem. longicornis and Haem. qinghaiensis can transmit Theileria luwenshuni and Theileria uilenbergi; Haem. longicornis can also transmit $T$. orientalis; and $T$. sinensis can be transmitted by Haem. qinghaiensis and Haem. japonica $[6,11,12]$. The geographical distribution of tick species linked to ovine and bovine Theileria spp. in China is listed in Fig. 1. 


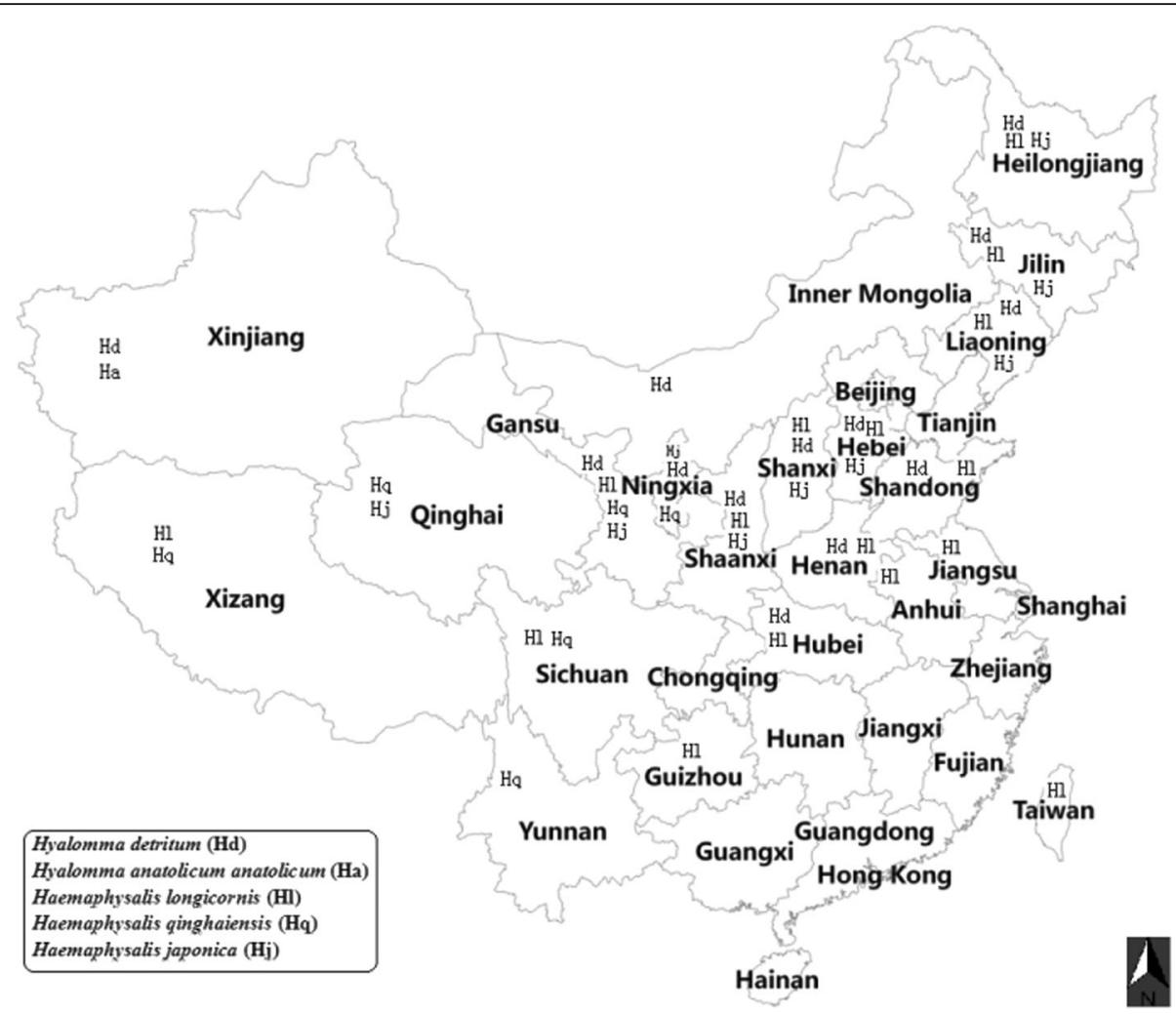

Fig. 1 Geographical distribution of various kinds of ticks related to bovine and ovine Theileria spp. in China

In the early phase, bovine theileriosis can be diagnosed based on observing ticks feeding on cattle, superficial lymph node enlargement, Theileria schizonts in lymphocytes with microscopic examination, and other clinical symptoms [3, 9]. Subsequently, Theileria parasites can be detected and identified in animals and ticks by polymerase chain reaction $(\mathrm{PCR})[13,14]$, reverse line blot (RLB) hybridization assay [15, 16], and loop-mediated isothermal amplification (LAMP) [17, 18]. Serodiagnosis of bovine theileriosis is performed by an indirect fluorescent antibody test (IFAT) [19-21]. Some enzyme-linked immunosorbent assays (ELISAs) to detect circulating antibodies against $T$. annulata were developed based on recombinant protein $T$. annulata surface protein (TaSP) or Tams1 [22-24]. At present, the ELISA method is widely applied as a cheap, fast and high-throughput method to screen and diagnose large numbers of clinical and field specimens.

T. luwenshuni surface protein (TISP) is a transmembrane protein that contains eight antigen peptides [21-23]. It shows that TISP owns some candidate epitopes. TISP shares a high similarity with TaSP [25-27]. TaSP is identified as an immunodominant antigen, and it successfully used to develop and to validate a recombinant-proteinbased ELISA for detecting the circulating antibodies of $T$. annulata-infected animals [22, 23, 28]. So, we concluded that we could use recombinant TISP (rTISP) as a diagnostic antigen in the ELISA. At present, serological epidemiological data about bovine theileriosis are deficient because of a lack of commercial or mature ELISA kits in China. Therefore, the objectives of this study were to develop an iELISA based on rTISP, and to perform a prevalence study of bovine theileriosis with the iELISA and microscopic examination in northern China.

\section{Methods}

\section{Preparation of recombinant TISP}

The functional fragment gene of TlSP was obtained from the merozoites cDNA library of $T$. luwenshuni using primers TISP-F (5'-GGA ATT CGA TCG ACA ACG GAA TCC T-3') and TISP-R (5'-CCA AGC TTT AAC CCG TCA GAG TCA T-3') [25]. The TISP gene was inserted and expressed in the pET30a vector. The expression of rTISP was verified by Western Blotting using anti-histidine antibody, and rTISP was purified using the BugBuster Ni-NTA His•Bind Purification Kit (Invitrogen, Carlsbad, CA, USA).

\section{Western blotting}

rTISP was separated using 12\% SDS-PAGE under reducing conditions and transferred to nitrocellulose membranes of $0.45 \mathrm{~mm}$ pore size (Amersham, Piscataway, 
USA). The nitrocellulose membranes were cut into $0.25 \mathrm{~cm}$ wide strips and blocked with $0.1 \mathrm{M}$ phosphatebuffered saline ( $\mathrm{pH}$ 7.6) containing 5\% skimmed milk powder and $0.1 \%$ Tween 20 (PBST) at $37{ }^{\circ} \mathrm{C}$ for $1 \mathrm{~h}$. A total of 12 different pathogen-positive sera ( $T$. luwenshuni, T. uilenbergi, T. ovis, T. annulata, T. orientalis, $T$. sinensis, Babesia bovis, B. major, B. bigemina, Anaplasma marginale, Brucella abortus, and Bovine epizootic fever virus) were used to check the antigenicity of rTISP. Antisera were diluted 1:100 and the secondary antibody (Anti-Bovine IgG; Sigma, St Louis, MO, USA) was diluted 1:20,000 in PBST. After three washes with PBST, positive signals were revealed by 5-bromo-4chloro-3-indolyl phosphate/nitro blue tetrazolium (BCIP/NBT) liquid substrate system (KPL, Gaithersburg, $\mathrm{MD}$, USA) for $2 \mathrm{~min}$.

\section{Sera}

Ninety-two negative sera (negative control) were collected from Chinese Holstein cattle at Lanzhou Dairy Farm, which is located in Lanzhou City, and neither ticks nor bovine theileriosis have been detected in the region for many years. Meanwhile, the whole blood samples with anticoagulant were collected and their smears were prepared. Ninety-two blood smears were negative by microscopic examination (ME); their relative sera were also negative by IFAT; and genomic DNA from 92 blood samples was negative by PCR [29]. As the 736 positive sera by IFAT (including 90 positive sera only by IFAT, and 646 positive sera by IFAT, PCR and ME), they were randomly picked from samples which were collected from local cattle in Xinjiang, an epidemic area of bovine theileriosis. Positive $(n=736)$ and negative $(n=92)$ sera were used in the next methods. The mixture of all positive sera was used as the positive control and the mixture of all negative sera was used as the negative control in the ELISA.

There are an estimated 30 million cattle in the study region [30]. The number of samples was calculated and collected according to twenty percent of the cattle population in selected sites, where the blood samples were permitted to be collected. A total of 2005 blood samples (including sera and whole blood with anticoagulant) were collected in 2011, 2012 and 2013 from 11 different provinces of northern China (Fig. 2): Xinjiang, Tibet, Qinghai, Gansu, Inner Mongolia, Shaanxi, Hebei, Henan, Shandong, Jilin, and Liaoning, most of which are epidemic areas for bovine theileriosis. The blood smears were prepared from peripheral blood and examined by microscopy. All of the serum samples were prepared and detected with iELISA, and the genomic DNA of all whole blood samples was extracted and detected by molecular methods. All sera were divided into five equal parts. These sera and their relative genomic DNA were

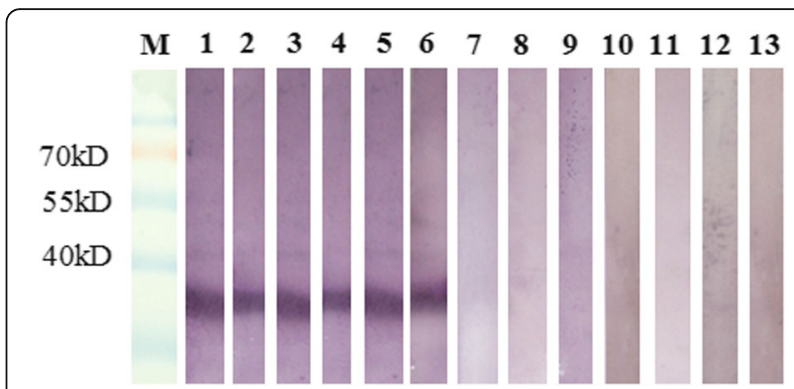

Fig. 2 Western blotting of rTISP. Lane M: prestained markers; Lane 1: T. luwenshuni; Lane 2: T. uilenbergi; Lane 3: T. ovis; Lane 4: T. annulata; Lane 5: T. orientalis; Lane 6: T. sinensis; Lane 7: B. bovis; Lane 8: B. major; Lane 9: B. bigemina; Lane 10: A. marginale; Lane 11: Br. abortus; Lane 12: Bovine epizootic fever virus; Lane 13: PBS

preserved at $-20{ }^{\circ} \mathrm{C}$ until utilizing them at Lanzhou Veterinary Research Institute.

\section{Development of ELISA and detection of samples}

A previously described ELISA protocol [31] was performed with some modifications. Briefly, 96-well microplates (Nunc, Roskilde, Denmark) were coated with $100 \mu \mathrm{l} /$ well rTISP $(5 \mu \mathrm{g} / \mathrm{ml})$ in a coating buffer $(0.1 \mathrm{M}$ carbonate-bicarbonate buffer, $\mathrm{pH} 9.6)$ at $4{ }^{\circ} \mathrm{C}$ overnight. After incubation with a blocking solution (2\% gelatin in PBST), the samples, blanks (PBST), standard positive and negative controls (diluted 1:100 in PBST) were distributed in duplicate. The plate was incubated with a peroxidase conjugate of monoclonal anti-bovine horseradish peroxidase IgG (Sigma, St Louis, MO, USA) (diluted 1:20,000 in PBST) at $37^{\circ} \mathrm{C}$ for $1 \mathrm{~h}$. After a washing step, $50 \mu \mathrm{l}$ of O-Phenylenediamine (OPD) (Sigma Aldrich, St Louis, MO, USA) was added to each well and incubated at room temperature for $10 \mathrm{~min}$. The reaction was stopped by adding $50 \mu \mathrm{l} /$ well of $2 \mathrm{M} \mathrm{H}_{2} \mathrm{SO}_{4}$. The optical density (OD) was measured with an ELISA reader (Microplate reader Model 680, Bio-Rad, Hercules, USA) at a wavelength of $490 \mathrm{~nm}$. The results are expressed as the percentage of the specific mean antibody rate $(\mathrm{AbR} \%)$, determined using the formula: AbR\% $=($ Sample mean OD - Negative control mean OD $) /$ (Positive control mean OD - Negative control mean OD) $\times 100 \%$. All sera samples were measured by ELISA.

\section{Detection of samples by microscopic examination}

Thin blood smears were prepared from peripheral blood of cattle. The smears were air-dried, fixed in methanol, stained with a $10 \%$ solution of Giemsa in PBS ( $\mathrm{pH} 7.2)$, and then subjected to microscopic analysis. One hundred fields per slide were searched for the presence of Theileria piroplasms. 


\section{Results}

\section{Cross-reactivity with hemoparasite sera}

The weight of recombinant TlSP was $\sim 38 \mathrm{kDa}$. Western blotting of rTlSP confirmed that there was specific recognition of the recombinant antigen rTISP by the positive sera of $T$. luwenshuni, $T$. uilenbergi, $T$. ovis, $T$. annulata, T. orientalis and T. sinensis; but there was no specific recognition of rTlSP with the positive sera of $B$. bovis, B. bigemina, B. major, A. marginale, Br. abortus and Bovine epizootic fever virus (Fig. 2).

\section{Cut-off, sensitivity and specificity of iELISA}

The cut-off, sensitivity and specificity were determined by 736 positive sera and 92 negative sera. The AbR\% was calculated for each serum sample, then the receiver operating characteristic (ROC) plots (including area under the curve; AUC), cut-off and Youden's index were evaluated with MedCalc statistical software (www.medcalc.org). Finally, the cut-off was determined to be $24.6 \%$, corresponding to $98.9 \%$ specificity $(95 \%$ confidence interval $[\mathrm{CI}]:$ 94.1-100\%) and $98.5 \%$ sensitivity (95\% CI: 97.3-99.3\%) (Fig. 3); AUC 0.996, and the Youden index J 0.9742 (Table 1).

\section{Serological epidemiology}

The prevalence of bovine theileriosis in 11 provinces is listed in Table 2 with ELISA and microscopic examination. The epidemiology results showed that the average sera-positive rate of bovine theileriosis was $95.4 \%$ by ELISA, the highest rate was $98.3 \%$ in Xinjiang Province, and the lowest rate was $84.1 \%$ in Qinhai Province. The highest prevalence was $38.9 \%$ in Shannxi Province by microscopic examination, the lowest was $5.1 \%$ in Tibet Province, and the average was $13.7 \%$.

\section{Discussion}

Serological surveillance of bovine theileriosis has been conducted in many countries [19, 20, 22-24, 32-35]. However, there is no previous report from China

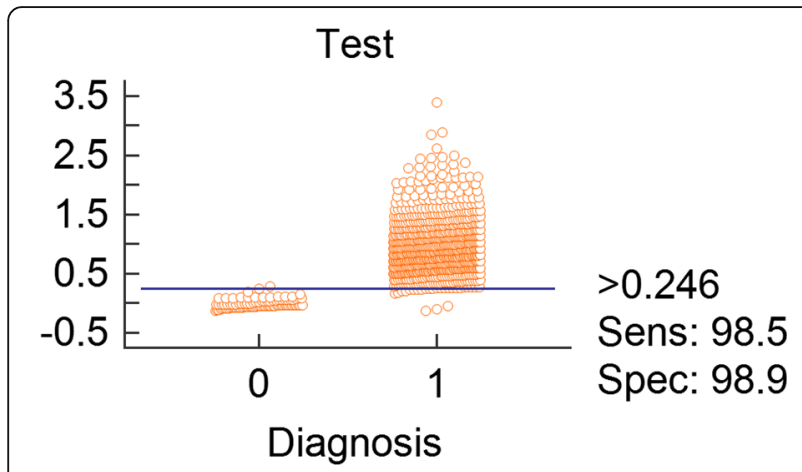

Fig. 3 Evaluation of the positive threshold value using mean AbR of negative and positive sera ( 0 = negative sera; 1 = positive sera)
Table 1 Area under the ROC curve (AUC) and Youden index of negative sera and positive sera

\begin{tabular}{ll}
\hline Parameter & Value \\
\hline Area under the ROC curve (AUC) & 0.996 \\
Standard Error & 0.00214 \\
$95 \%$ Confidence Interval & $0.989-0.999$ \\
$Z$ statistic & 232.120 \\
Significance level P (Area = 0.5) & $<0.0001$ \\
Youden index J & 0.9742 \\
Associated criterion & $>0.2460$
\end{tabular}

because of the lack of domestic commercial or mature ELISA kits that can detect bovine Theileria antibodies. In addition, the foreign ELISA kits are more expensive. Compared to other methods, the advantages of ELISA are that they are less laborious and easier to perform, and a large number of samples can be detected and screened in a short time. In addition, compared to ELISA using crude antigens (such as whole parasite antigens), recombinant-protein-based ELISAs reduce false results. Serological assays are more suitable for diagnosis in the mid and late phase of theileriosis and in carrier animals (e.g. cattle, buffalo and yaks) in which the antibody titers against Theileria parasites are usually higher but the piroplasm parasitemia maybe drop to undetectable levels with microscopic examination [22].

The ELISA based on rTISP has proven to be a highly specific and sensitive assay for detecting the circulating antibodies against Theileria spp. in China. Three bovine Theileria spp. have been reported in China [3, 5, 6, 12], and their specific positive sera against each pathogen

Table 2 Prevalence of bovine theileriosis in cattle in northern China determined by iELISA and microscopic examination

\begin{tabular}{lllll}
\hline Province & $\begin{array}{l}\text { No. of } \\
\text { samples }\end{array}$ & $\begin{array}{l}\text { No. of } \\
\text { positive sera } \\
\text { by ELISA }\end{array}$ & $\begin{array}{l}\text { Percentage } \\
\text { of positive } \\
\text { sera (\%) }\end{array}$ & $\begin{array}{l}\text { Percentage of } \\
\text { positive blood } \\
\text { smears (\%) }\end{array}$ \\
\hline Xinjiang & 180 & 177 & 98.3 & 30.0 \\
Gansu & 584 & 527 & 90.2 & 7.0 \\
Liaoning & 111 & 109 & 98.2 & 27.0 \\
Jilin & 25 & 24 & 96.0 & 12.0 \\
Shaanxi & 54 & 53 & 98.1 & 38.9 \\
Qinghai & 270 & 227 & 84.1 & 18.1 \\
Inner Mongolia & 316 & 301 & 98.1 & 10.0 \\
Hebei & 30 & 29 & 96.6 & 13.3 \\
Henan & 60 & 57 & 95.0 & 30.0 \\
Tibet & 315 & 309 & 98.1 & 5.1 \\
Shandong & 60 & 58 & 96.6 & 10.0 \\
Total & 2005 & 1871 & & 13.7 \\
Average & & & 95.4 & \\
\hline & & & & \\
\hline
\end{tabular}


were analyzed with Western Blotting and ELISA in this study; all of them cross-reacted with rTISP. As a comparison, there was no cross-reactivity between rTISP and positive sera of B. bovis, B. major, B. bigemina, A. marginale, Br. abortus and Bovine epizootic fever virus in Western Blotting.

We chose 736 positive samples to calculate the sensitivity and specificity accurately. They included the strong positive samples (646 positive sera by IFAT, PCR and ME) and the faint positives samples (90 positive sera only by IFAT). The employ of weak positive specimen makes the cut-off value to be reasonable and can avoid false negative results.

The cut-off value resulting in both maximal sensitivity and specificity was determined by two-graph ROC analysis, ROC plots efficiency, Youden's index and likelihood ratios [36]. The data showed that the AUC was 0.996 in ROC plots, which demonstrated the accuracy of the index. The test was reliable, and the indexes are the best performance because the area is closer to 1 [37]. Moreover, AUC is a quantitative, descriptive expression of how close the ROC curve is to the perfect one (AUC =1.0) [38]. Youden index J was 0.9742, which indicated that, unusually, there were neither false-positive nor false-negative results from the test [39]. Compared with the positive rate with microscopy, which was $<40 \%$ at the highest, iELISA based on rTISP is suitable for detecting antibodies against bovine Theileria spp. Therefore, the method can estimate whether the local cattle had been infected by Theileria spp. before the cattle were detected. It remains to be detected by blood smears and molecular methods, such as PCR, RLB and LAMP, to diagnose whether the cattle are infected by Theileria spp. After the cattle infected with Theileria parasites recovered, most remained in a state of low parasitemia or carrier state for 3-6 months (data unpublished). Investigation of bovine theileriosis is of veterinary significance.

The epidemiological survey indicated that all the provinces investigated had a high positive rate from 84.1 to 98.3\% with iELISA. In comparison, the positive rate was $5.1-38.9 \%$ by microscopic examination. Microscopic examination can diagnose Theileria parasites in the early and middle phase of theileriosis, whereas, in the later phase and carrier state, parasitemia tends to a low level so that Theileria can not be detected by microscopic examination. For example, in Tibet, the positive rate was $5.1 \%$ by microscopic examination, but up to $98.1 \%$ by ELISA.

\section{Conclusions}

The epidemiological data showed that bovine theileriosis was widespread in northern China. Therefore, some efficient measures should be implemented for calves and exotic cattle in northern China.

\section{Abbreviations}

BCIP/NBT: 5-bromo-4-chloro-3-indolyl phosphate/nitro blue tetrazolium;

Cl: Confidence interval; iELISA: Indirect enzyme-linked immunosorbent assay; IFAT: Indirect fluorescent antibody test; OD: Optical density; OPD: O-

Phenylenediamine; PBST: Phosphate-buffered saline (PBS) containing $0.1 \%$ Tween 20; ROC: Receiver operating characteristic; rTISP: Recombinant TISP; TISP: Theileria luwenshuni surface protein

\section{Acknowledgments}

We thank International Science Editing for help with modifying the paper.

\section{Funding}

This study was financially supported by the NSFC (Nos. 31272556, 31372432, 31101621, 31201899), NBCIS (CARS-38), ASTIP, FRIP (2014ZL010), CAAS, Creative Research Groups of Gansu Province (No. 1210RJIA006), '948'(2014-S05), Special Fund for Agro-scientific Research in the Public Research (Nos. 201303035, 201303037), MOA; 973 Program (2010CB530206), Basic Research program (CRP No. 16198/R0), Supporting Program (2013BAD12B03, 2013BAD12B05), Specific Fund for Sino-Europe Cooperation, MOST, China, State Key Laboratory of Veterinary Etiological Biology Project. The research was also facilitated by CRP No. 16198/RO IAEA, and PIROVAC (KBBE-3-245145) of the European Commission.

\section{Availability of data and material}

The dataset supporting the conclusions of this article is included within the article.

\section{Authors' contributions}

LYa and LYo conceived the study and all authors participated in its design and coordinated the experiments. LYa, LQ and YJ performed the Western Blotting. LYa and LQ developed the protocol for the iELISA. YJ, GP and LJ collected the serum samples. LG and GG processed the serum samples. LYo, $\mathrm{LZ}$ and $\mathrm{YH}$ performed the microscopic examination. All authors have read and approved the final manuscript.

\section{Competing interests}

The authors declare that they have no competing interests.

\section{Consent for publication}

Not applicable.

\section{Ethics approval and consent to participate}

This study was approved by the Animal Ethics Committee, Lanzhou Veterinary Research Institute, Chinese Academy of Agricultural Sciences. All cattle were handled in accordance with good animal practices required by the Animal Ethics Procedures and Guidelines of the People's Republic of China.

\section{Author details}

${ }^{1}$ State Key Laboratory of Veterinary Etiological Biology, Key Laboratory of Veterinary Parasitology of Gansu Province, Lanzhou Veterinary Research Institute, Chinese Academy of Agricultural Science, Xujiaping 1, Lanzhou, Gansu 730046, People's Republic of China. ${ }^{2}$ Jiangsu Co-innovation Center for Prevention and Control of Important Animal Infectious Diseases and Zoonoses, Yangzhou 225009, People's Republic of China.

Received: 22 February 2016 Accepted: 9 November 2016

Published online: 18 November 2016

\section{References}

1. Simuunza M, Weir W, Courcier E, Tait A, Shiels B. Epidemiological analysis of tick-borne diseases in Zambia. Vet Parasitol. 2011;175:331-42.

2. Aktas M, Dumanli N, Angin M. Cattle infestation by Hyalomma ticks and prevalence of Theileria in Hyalomma species in the east of Turkey. Vet Parasitol. 2004;119(1):1-8.

3. Luo J, Lu W. Cattle theileriosis in China. Trop Anim Health Prod. 1997;29(4 Suppl):4S-7S.

4. Marcelino I, de Almeida AM, Ventosa M, Pruneau L, Meyer DF, Martinez D, et al. Tick-borne diseases in cattle: applications of proteomics to develop new generation vaccines. J Proteomics. 2012;75:4232-50.

5. Bai Q, Liu G, Hen G. An unidentified species of Theileria infective for cattle discovered in China. Trop Anim Health Prod. 1997;29:43S-7S. 
6. Bai Q, Liu GY, Yin H, Zhao QZ, Liu DK, Ren JX, et al. Theileria sinensis sp nov: a new species of Bovine Theileria-classical taxonomic studies. Acta Veterinaria et Zootechnica Sinica. 2002;33:73-7 (in Chinese).

7. Yin H, Guan GQ, Ma ML, Luo JX, Lu BY, Yuan GL, et al. Haemaphysalis qinghaiensis ticks transmit at least two different Theileria species: one is infective to yaks, one is infective to sheep. Vet Parasitol. 2002;107:29-35.

8. Teng KF, Jiang ZJ. Economic insect fauna of China. Fasc 39, Acari: Ixodidae. Bejing: Science Press; 1991. p. 158-81.

9. Yin H, Luo JX, Lu WS. Control of tropical theileriosis with attenuated schizont vaccine in China. Vaccine. 2008;26 Suppl 6:G11-3.

10. Li Y, Guan G, Liu A, Peng Y, Luo J, Yin H. Experimental transmission of Theileria ovis by Hyalomma anatolicum anatolicum. Parasitol Res. 2010;106(4): 991-4.

11. Li Y, Luo J, Guan G, Ma M, Liu A, Liu J, et al. Experimental transmission of Theileria uilenbergi infective for small ruminants by Haemaphysalis longicornis and Haemaphysalis qinghaiensis. Parasitol Res. 2009;104(5):1227-31.

12. Yin H, Luo J, Schnittger L, Lu B, Beyer D, Ma M, et al. Phylogenetic analysis of Theileria species transmitted by Haemaphysalis qinghaiensis. Parasitol Res. 2004:92(1):36-42.

13. d'Oliveira C, van der Weide M, Habela MA, Jacquiet $P$, Jongejan F. Detection of Theileria annulata in blood samples of carrier cattle by PCR. J Clin Microbiol. 1995;33:2665-9.

14. Aktas M, Dumanli N, Cetinkaya B, Cakmak A. Field evaluation of PCR in detecting Theileria annulata infection in cattle in eastern Turkey. Vet Rec. 2002;150(17):548-9.

15. Schnittger L, Yin H, Bai Q, Gubbels MJ, Beyer D, Niemann S, et al. Simultaneous detection and differentiation of Theileria and Babesia parasites infecting small ruminants by reverse line blotting. Parasitol Res. 2004;2:189-96.

16. Altay K, Aydin MF, Dumanli N, Aktas M. Molecular detection of Theileria and Babesia infections in cattle. Vet Parasitol. 2008;158(4):295-301.

17. Liu AH, Guan GQ, Du PF, Liu ZJ, Gou HT, Liu JL, et al. Loop-mediated isothermal amplification (LAMP) assays for the detection of Theileria annulata infection in China targeting the 18S rRNA and ITS sequences. Exp Parasitol. 2012;131:125-9.

18. Liu AH, Guan GQ, Du PF, Gou HT, Liu ZJ, Liu JL, et al. Rapid identification and differentiation of Theileria sergenti and Theileria sinensis using a loop-mediated isothermal amplification (LAMP) assay. Vet Parasitol. 2013;191:15-22.

19. Burridge MJ, Kimber CD. The indirect fluorescent antibody test for experimental East Coast fever (Theileria parva infection of cattle). Evaluation of a cell culture schizont antigen. Res Vet Sci. 1972;13(5):451-5.

20. Kimber CD, Burridge MJ. The indirect fluorescent antibody test for experimental East Coast fever (Theileria parva infection of cattle). Evaluation of dried blood samples as a source of antibody. Res Vet Sci. 1972;13(2):133-5.

21. OIE. Manual of diagnostic tests and vaccines for terrestrial animals, vol. 1-2. 7th ed. Paris: Office International Des Epizooties; 2014.

22. Bakheit MA, Schnittger L, Salih DA, Boguslawski K, Beyer D, Fadl M, et al. Application of the recombinant Theileria annulata surface protein in an in indirect ELISA for the diagnosis of tropical theileriosis. Parasitol Res. 2004;92: 299-302.

23. Seitzer U, Bakheit MA, Salih DE, Ali A, Haller D, Yin H, et al. From molecule to diagnostic tool: Theileria annulata surface protein TaSP. Parasitol Res. 2007;101:S217-23.

24. Rajendran C, Ray DD. Diagnosis of tropical bovine theileriosis by ELISA with recombinant merozoite surface protein of Theileria annulata (Tams1). J Parasit Dis. 2014;38:41-5.

25. He H, Li Y, Liu Z, Yang J, Chen Z, Luo J, et al. Cloning and Prokaryotic expression of TISP gene of Theileria luwenshuni. Chin Vet Sci. 2012;42:1139-43 (in Chinese).

26. Li Y, Liu Z, Chen Z, Liu J, Yang J, He H, et al. An ELISA method for detection of ovine and caprine theileriosis. Patent. 2015. Patent Number: ZL20121012339.8

27. He H, Li Y, Liu J, Liu Z, Yang J, Liu A, et al. An indirect ELISA for detection of Theileria spp. antibodies using a recombinant protein (rTISP) from Theileria luwenshuni. Exp Parasitol. 2016;166:89-93.

28. Renneker S, Kullmann B, Gerber S, Dobschanski J, Bakheit MA, Geysen D, et al. Development of a competitive ELISA for detection of Theileria annulata infection. Transbound Emerg Dis. 2008;55:249-56.

29. Allsopp BA, Baylis HA, Allsopp MT, Cavalier-Smith T, Bishop RP, Carrington DM, et al. Discrimination between six species of Theileria using oligonucleotide probes which detect small subunit ribosomal RNA sequences. Parasitology. 1993;107:157-65.
30. Cao BH. China bull map (the Second Edition). Yak beef cattle industry technology research and development center. 2011; Censor code: GS $(2008,1361)$

31. Guan GQ, Chauvin A, Rogniaux H, Luo JX, Yin H, Moreau E. Merozoite proteins from Babesia sp. BQ1 (Lintan) as potential antigens for serodiagnosis by ELISA. Parasitol. 2010;137:927-38.

32. Gubbels MJ, d'Oliveira C, Jongejan F. Development of an indirect Tams1 enzyme-linked immunosorbent assay for diagnosis of Theileria annulata infection in cattle. Clin Diagn Lab Immunol. 2000;7:404-11.

33. Renneker S, Abdo J, Ahmed JS, Seitzer U. Field validation of a competitive ELISA for detection of Theileria annulata infection. Parasitol Res. 2009;106(1): 47-53.

34. Billiouw M, Brandt J, Vercruysse J, Speybroeck N, Marcotty T, Mulumba M, et al Evaluation of the indirect fluorescent antibody test as a diagnostic tool for East Coast fever in eastern Zambia. Vet Parasitol. 2005:127(3-4):189-98.

35. Yin H, Lu W, Luo J. Babesiosis in China. Trop Anim Health Prod. 1997;29: 11S-5S.

36. Greiner M, Sohr D, Goble P. A modified ROC analysis for the selection of cut-off values and the definition of intermediate results of serodiagnostic test. J Immunol Methods. 1995;185:123-32.

37. Mboloi MM, Bekker CP, Kruitwagen C, Greiner M, Jongejan F. Validation of the indirect MAP1-B enzyme-linked immunosorbent assay for diagnosis of experimental Cowdria ruminantium infection in small ruminants. Clin Diagn Lab Immunol. 1999;6:66-72.

38. Greiner M. Two-graph receiver operating characteristic (TG-ROC): a Microsoft-EXCLE template for the selection of cut-off values in diagnostic test. J Immunol Methods. 1995;185:145-6.

39. Youden WJ. Index for rating diagnostic tests. Cancer. 1950;3:32-5.

\section{Submit your next manuscript to BioMed Central and we will help you at every step:}

- We accept pre-submission inquiries

- Our selector tool helps you to find the most relevant journal

- We provide round the clock customer support

- Convenient online submission

- Thorough peer review

- Inclusion in PubMed and all major indexing services

- Maximum visibility for your research

Submit your manuscript at www.biomedcentral.com/submit
) Biomed Central 